\title{
Efficacy and safety of ERCP in elderly patients with an ECOG performance status of 3-4
}

\author{
SHOHEI ASADA ${ }^{1}$, AKITOSHI DOUHARA ${ }^{1}$, HIROTSUGU UENO ${ }^{1}$, \\ KOJI MURATA $^{1}$, KOJI YANASE ${ }^{1}$ and HITOSHI YOSHIJI ${ }^{2}$ \\ ${ }^{1}$ Department of Gastroenterology, Saiseikai Chuwa Hospital, Sakurai, Nara 633-0054; \\ ${ }^{2}$ Third Department of Internal Medicine, Nara Medical University, Kashihara, Nara 634-8522, Japan
}

Received November 14, 2019; Accepted January 29, 2020

DOI: $10.3892 /$ wasj.2020.36

\begin{abstract}
The aim of the present study was to clarify the efficacy and safety of endoscopic retrograde cholangiopancreatography (ERCP) for elderly patients with a performance status (PS) of 3-4, which is the highest risk group. The records of patients aged over 75 years of age, who underwent ERCP at Saiseikai Chuwa Hospital from April, 2017 to April, 2018, were retrospectively analyzed. These patients were divided into the PS 0-2 group and PS 3-4 group. For both groups, clinical characteristics, such as age, sex, PS, the prevalence rate of moderate or severe cholangitis, Charlson comorbidity index (CCI), technical success rate, adverse events and fatal cases within 30 days following ERCP were analyzed. The results revealed that in total, 94 patients (158 procedures) aged over 75 years underwent ERCPs; 73 patients (116 procedures) were in the PS 0-2 group, and 21 patients (42 procedures) were in the PS 3-4 group. The average age did not differ significantly between the groups. The prevalence rates of moderate or severe cholangitis and CCI were higher in the PS 3-4 group. The technical success rate was high in both groups. Although the rate of adverse events did not differ between the 2 groups, 2 fatal cases occurred during the 30 days following ERCP in the PS 3-4 group. In each case, anorexia and an influenza infection led to a fatal outcome. Overall, this study demonstrates that ERCP for elderly patients with a PS of 3-4 is effective and safe. However, attention should be paid to ERCP in patients
\end{abstract}

Correspondence to: Dr Akitoshi Douhara, Department of Gastroenterology, Saiseikai Chuwa Hospital, 323 Abe, Sakurai, Nara 633-0054, Japan

E-mail: aki-do@hotmail.co.jp

Abbreviations: AST, aspartate aminotransferase; ALT, alanine aminotransferase; ERCP, endoscopic retrograde cholangiopancreatography; EBS, endoscopic biliary stent; PS, performance status; CCI, Charlson comorbidity index; ECOG, Eastern Cooperative Oncology Group; DASI, Duke Activity Status Index; SEMS, self-expandable metallic stent

Key words: ERCP, elderly, performance status, comorbidity with a PS of 3-4, as it may result in a fatal outcome caused by incidents unrelated to the ERCP procedure.

\section{Introduction}

Endoscopic retrograde cholangiopancreatography (ERCP) is widely performed as the diagnostic and therapeutic tool intended for patients with pancreato-biliary diseases. The frequency of ERCP for elderly patients is increasing in accordance with the overall aging of the population worldwide (1). To date, ERCP has been known to be associated with severe adverse events, such as pancreatitis, gastrointestinal bleeding and perforation (1). Although it has been reported that ERCP can be safely performed in elderly patients (2-6), the clinical status of these patients varies due to comorbidities, nutritional status, cognition, etc., which causes endoscopists to debate whether or not ERCP should be performed. The Eastern Cooperative Oncology Group (ECOG) scale of performance status (PS) is widely used for the quantification of the general well-being and daily life activities of patients (7). Elderly patients with a decreased PS are a high-risk group, and the efficacy and safety of ERCP for these patients is unclear. Thus, the aim of this study was to elucidate the efficacy and safety of ERCP in elderly patients with a PS of 3-4, compared to those with a PS of 0-2, hospitalized in Saiseikai Chuwa Hospital.

\section{Patients and methods}

Study design. The present study was conducted at Saiseikai Chuwa Hospital, Sakurai, Nara, Japan. This hospital is located in a rural district in Nara, and the average age of patients in this area is very high. The records of the patients, who underwent ERCP in this facility from April, 2017 to April, 2018 , were retrospectively analyzed. In total, 146 patients (232 procedures) underwent ERCP, patients aged $<75$ years were excluded, and 94 patients (158 procedures) were enrolled in this study. The patients were divided into the PS 0-2 group and PS 3-4 group. For both groups, clinical characteristics were analyzed, such as age, sex, PS, primary disease for ERCP, the prevalence rate of moderate or severe cholangitis [assessed by the severity assessment of acute cholangitis per the Tokyo Guidelines 13 (TG13) (8)], the use of antithrombotic drugs, history of gastrectomy and the Charlson comorbidity index 
(CCI) (9), which is a weighted index that assigns a different risk score (from 1 to 6 ) to 22 conditions and their related relative risk of 1-year mortality. Clinical outcomes were analyzed, such as performed procedures, the number of ERCPs for each patient, the technical success rate, adverse events caused by ERCPs, admission periods and fatal cases occurred within 30 days following ERCP.

Procedures. In the facility, 5 gastroendoscopists performed ERCP. All ERCP procedures were performed by one operator and at least one assistant among 5 gastroendoscopists. A rule was established to complete the ERCP procedures within $1 \mathrm{~h}$ from scope insertion for all patients, as the ERCP procedure bears the risk of adverse events, such as pancreatitis, even when performed by endoscopic specialists, and it is estimated that a lengthy procedure bears the risk of adverse events following ERCP. According to the TG13 flowchart (10), not all procedures are performed in a single session for patients with moderate or severe cholangitis. In the first session, only endoscopic drainage was performed, followed, after the improvement of cholangitis, by other procedures such as endoscopic lithotomy. The TJF-260V (Olympus Medical Systems Co.) and ED-450XL8/B (FUJIFILM Co.) endoscopes were used, and for patients who had Billroth II or Roux-en Y reconstructions, the SIF-H290S (Olympus Medical Systems Co.) was used, which is a short-type single-balloon-assisted endoscope useful for ERCP in patients with altered gastrointestinal anatomy (11). For all procedures, carbon dioxide $\left(\mathrm{CO}_{2}\right)$ insufflation was used during ERCP to reduce post-procedural pain and abdominal distension $(12,13)$. For the purposes of sedation during ERCP, midazolam and pentazocine were used, monitoring vital signs and the degree of sedation, all the while increasing the sedation if necessary.

Definition. The PS score (7) was labeled according to information on the general status prior to admission.

Severity assessment of cholangitis. The severity of cholangitis was assessed by the severity assessment of acute cholangitis as per the Tokyo Guidelines 2013 (8). The adverse events caused by ERCP procedures are defined as follows: i) Pancreatitis: The onset of new abdominal pain with at an least 3-fold elevation of serum amylase levels following ERCP; ii) hyperamylasemia: At least a 3-fold elevation of serum amylase levels following ERCP, without abdominal pain; and iii) biliary infection, including cholangitis, cholecystitis and liver abscess. The onset of the adverse events was evidenced after the ERCP procedures and did not exist before the ERCP procedures. Liver dysfunction is defined as the elevation of L-aspartate aminotransferase (AST) and L-alanine aminotransferase (ALT) that were at normal levels before the ERCP procedures and were found to be above normal levels after the ERCP procedures in the absence of any other cause. Mallory-Weiss syndrome is defined as the laceration of gastroesophageal junction caused by nausea or vomiting with or following ERCP procedures.

Statistical analysis. Comparisons between the study groups were performed with the Wilcoxon signed-rank test for quantitative variables and the Chi-square test for qualitative variables. A P-value $<0.05$ was considered to indicate a statistically significant difference. All statistical analyses were performed using IBM SPSS statistics version 22 (IBM Corp.).

\section{Results}

Clinical characteristics. The clinical characteristics of the patients in both groups are presented in Table I. A total of 94 patients (158 procedures) who were over 75 years old and underwent ERCP were divided into 2 groups as follows: The PS 0-2 group with 73 patients (116 procedures), and the PS 3-4 group with 21 patients (42 procedures). The average age did not differ significantly between the 2 groups. The ERCP rate in females was significantly higher in the PS 3-4 group than in the PS 0-2 group. The numbers of patients for each PS were 31 for PS 0, 36 for PS 1, 6 for PS 2, 4 for PS 3 and 17 for PS 4. Of note, the prevalence rates of moderate or severe cholangitis and the CCI were significantly higher in the PS 3-4 group than in the PS 0-2 group. The rate of anti-thrombotic drug use did not differ between the groups. In the PS 0-2 group, 9 patients had undergone gastrectomy (3 patients with Billroth I reconstruction, 3 patients with Billroth II reconstruction and 3 patients with Roux-en-Y reconstruction); however, in the PS 3-4 group, no patients had undergone gastrectomy. In both groups, no patients had undergone pancreaticoduodenectomy or choledochojejunostomy. The majority of the indications for ERCP in both groups were choledocholithiasis and malignant biliary obstruction (Table II).

ERCP results. The ERCP results for both groups are presented in Tables III-V. The performed procedures did not differ in either group. In particular, endoscopic biliary stent (EBS) and endoscopic lithotomy were frequently observed (Table III). Patients in the PS 3-4 group required frequent ERCP for one primary disease and longer admission periods (Table V). The reason was that patients in the PS 3-4 group had a higher prevalence rate of moderate or severe cholangitis (Table I). Only biliary drainage was performed for the first procedure, and other procedures such as endoscopic lithotomy were performed following the improvement of cholangitis. Moreover, a number of patients in the PS 3-4 group were often discharged to the nursing facility. Thus, several procedures and the adjustment of the discharge schedule required longer admission periods. Subsequently, the technical success rate for planned procedures was adequately high in both groups. There were no cases that required the suspension of ERCP procedures due to hypotension, bradycardia or respiratory depression caused by oversedation. In the PS 3-4 group, all planned procedures were completed (Table V). However, in the PS 0-2 group, 5 cases failed to achieve bile duct or pancreatic duct cannulation at the first ERCP. However, 3 cases were completely successful at the second ERCP, and 2 cases were changed to perform procedures under a non-endoscopic approach. One case after Roux-en-Y reconstruction failed to reach the papilla of Vater. Finally, the rate of adverse events caused by ERCP procedures did not differ significantly in both groups (Table IV). There were no severe adverse events, such as perforation and bleeding, which required endoscopic hemostasis or blood transfusion. Although 5 cases of post-ERCP-pancreatitis were observed only in the PS 0-2 group, they were improved only by conservative therapy. Despite the rate of adverse events by 
Table I. Baseline characteristics in elderly patients with both PS 0-2 and 3-4.

\begin{tabular}{lcccc}
\hline Variable & $\begin{array}{c}\text { All patients } \\
\text { (94 patients, } \\
158 \text { procedures) }\end{array}$ & $\begin{array}{c}\text { PS 0-2 group } \\
\text { (73 patients, } \\
116 \text { procedures) }\end{array}$ & $\begin{array}{c}\text { PS 3-4 group } \\
(21 \text { patients, } \\
42 \text { procedures) }\end{array}$ & P-value \\
\hline Age, median (range) & $84(75-98)$ & $84(75-96)$ & $86(78-98)$ & N.S. \\
Sex, rate of female (\%) & 52.1 & 43.8 & 80.9 & P=0.003 \\
Performance status, 0/1/2/3/4 & $31 / 36 / 6 / 4 / 17$ & $31 / 36 / 6 / 0 / 0$ & $0 / 0 / 0 / 4 / 17$ & - \\
Prevalence rate of moderate or severe cholangitis, $\mathrm{n}(\%)$ & $29(30.9)$ & $14(19.1)$ & $15(71.4)$ & $\mathrm{P}<0.001$ \\
Charlson comorbidity index, median (range) & $1(0-7)$ & $1(0-7)$ & $2(1-3)$ & $\mathrm{P}=0.001$ \\
Antithrombotic drug therapy, $\mathrm{n}(\%)$ & $34(36.2)$ & $28(38.4)$ & $6(28.6)$ & N.S. \\
Altered gastrointestinal anatomy, Billroth I, II, Roux-en-Y & $3 / 3 / 3$ & $3 / 3 / 3$ & $0 / 0 / 0$ & - \\
\hline
\end{tabular}

PS, performance status; N.S., not significant.

Table II. Indications for ERCP in elderly patients with both PS 0-2 and 3-4.

\begin{tabular}{lcccr}
\hline & $\begin{array}{c}\text { All patients } \\
\text { (94 patients, } \\
\text { 158 procedures) }\end{array}$ & $\begin{array}{c}\text { PS 0-2 group } \\
\text { (73 patients, } \\
116 \text { procedures) }\end{array}$ & $\begin{array}{c}\text { PS 3-4 group } \\
\text { (21 patients, } \\
\text { 42 procedures) }\end{array}$ & P-value \\
\hline Choledocholithiasis, $\mathrm{n}(\%)$ & $56(59.6)$ & $46(63.0)$ & $10(47.6)$ & N.S. \\
Malignant biliary obstruction, $\mathrm{n}(\%)$ & $32(34.0)$ & $24(32.9)$ & $8(38.1)$ & N.S. \\
Gallstone pancreatitis, $\mathrm{n}(\%)$ & $2(2.1)$ & $0(0.0)$ & $2(9.5)$ & N.S. \\
Lemmel syndrome, $\mathrm{n}(\%)$ & $1(1.1)$ & $0(0.0)$ & $1(4.8)$ & N.S. \\
Others, $\mathrm{n}(\%)$ & $3(3.2)$ & $3(4.1)$ & $0(0.0)$ & N.S. \\
\hline
\end{tabular}

ERCP, endoscopic retrograde cholangiopancreatography; PS, performance status; N.S., not significant.

Table III. ERCP procedures in elderly patients with both PS 0-2 and 3-4.

\begin{tabular}{lcccc}
\hline Variable & $\begin{array}{c}\text { All patients } \\
\text { (94 patients, } \\
158 \text { procedures })\end{array}$ & $\begin{array}{c}\text { PS 0-2 group } \\
(73 \text { patients, } \\
116 \text { procedures) }\end{array}$ & $\begin{array}{c}\text { PS 3-4 group } \\
\text { (21 patients, } \\
42 \text { procedures) }\end{array}$ & P-value \\
\hline Endoscopic biliary stent (EBS), $\mathrm{n}(\%)$ & $69(43.7)$ & $51(44.0)$ & $18(42.9)$ & N.S. \\
Endoscopic nasobiliary drainage (ENBD), $\mathrm{n}(\%)$ & $6(3.8)$ & $5(4.3)$ & $1(2.4)$ & - \\
Self expandable metalic stent (SEMS), $(\%)$ & $12(7.6)$ & $9(7.8)$ & $3(7.1)$ & - \\
Endoscopic sphincterotomy (EST), $\mathrm{n}(\%)$ & $55(34.8)$ & $40(34.5)$ & $15(35.7)$ & - \\
Endoscopic papillary large balloon dilation (EPLBD), $\mathrm{n}(\%)$ & $11(7.0)$ & $8(6.9)$ & $3(7.1)$ & - \\
Endoscopic lithotomy, $\mathrm{n}(\%)$ & $58(36.7)$ & $39(33.6)$ & $19(45.2)$ & - \\
SEMS cleaning, $\mathrm{n}(\%)$ & $2(1.3)$ & $1(0.9)$ & $1(2.4)$ & - \\
\hline
\end{tabular}

ERCP, endoscopic retrograde cholangiopancreatography; PS, performance status; N.S., not significant.

ERCP procedures not differing in both groups, there were 2 fatal cases during the 30 days following ERCP in the PS 3-4 group and no fatal cases in the PSO-2 group (Table V). The two fatal cases during 30 days following ERCP were the following.

i) Case A: An 86-year-old female with PS 4 underwent placement of a covered-type self-expandable metallic stent (SEMS) for biliary obstruction with pancreatic cancer. After 1 month of ERCP, she was admitted to the hospital for cholangitis due to SEMS obstruction. She then underwent SEMS cleaning and improved. However, she was readmitted for cholangitis due to a recurrence of SEMS obstruction (Fig. 1A). The prolapsed, obstructed SEMS (Fig. 1B) was removed and replaced by an EBS (Fig. 1C). Subsequently, although cholangitis was improved, her general condition gradually worsened due to anorexia caused by pancreatic cancer. As a result, she died 26 days after ERCP. 
Table IV. Post-ERCP complications in elderly patients with both PS 0-2 and 3-4.

\begin{tabular}{|c|c|c|c|c|}
\hline Variable & $\begin{array}{c}\text { All patients } \\
\text { (94 patients, } \\
158 \text { procedures) }\end{array}$ & $\begin{array}{c}\text { PS 0-2 group } \\
\text { (73 patients, } \\
116 \text { procedures) }\end{array}$ & $\begin{array}{l}\text { PS 3-4 group } \\
\text { (21 patients, } \\
42 \text { procedures) }\end{array}$ & P-value \\
\hline Total, n (\%) & $25(15.8)$ & $19(16.4)$ & $6(14.3)$ & N.S. \\
\hline Pancreatitis, n (\%) & $5(3.1)$ & $5(4.3)$ & $0(0.0)$ & - \\
\hline Hyperamylasemia, n (\%) & $14(8.9)$ & $10(9.5)$ & $4(9.5)$ & - \\
\hline Biliary infection, n (\%) & $2(1.3)$ & $1(0.9)$ & $1(2.4)$ & - \\
\hline Liver dysfunction, n (\%) & $3(1.9)$ & $3(2.6)$ & $0(0.0)$ & - \\
\hline Mallory-Weiss syndrome, n (\%) & $1(0.6)$ & $0(0.0)$ & $1(2.4)$ & - \\
\hline Perforation, n (\%) & $0(0.0)$ & $0(0.0)$ & $0(0.0)$ & - \\
\hline
\end{tabular}

ERCP, endoscopic retrograde cholangiopancreatography; PS, performance status; N.S., not significant.

Table V. Post-ERCP outcomes in elderly patients with both PS 0-2 and 3-4.

\begin{tabular}{lccc}
\hline & All patients & PS 0-2 group & PS 3-4 group \\
Variable & $\begin{array}{c}\text { (94 patients, } \\
\text { 158 procedures) }\end{array}$ & $\begin{array}{c}\text { (73 patients, } \\
116 \text { procedures) }\end{array}$ & $\begin{array}{c}\text { 42 procedures) } \\
\text { P-value }\end{array}$ \\
\hline Numbers of ERCP for one patient, average (range) & $1.7(1-4)$ & $1.6(1-4)$ & $2.0(1-4)$ \\
Admission period (days), median (range) & $17(4-98)$ & $15(4-80)$ & $23(10-98)$ \\
Technical Success Rate, $\%$ & 96.2 & 94.8 & 100 \\
Fatal case in 30 days following ERCP, $n$ & 2 & 0.003 & N.S. \\
\hline
\end{tabular}

ERCP, endoscopic retrograde cholangiopancreatography; PS, performance status; N.S., not significant.

ii) Case B: A 98-year-old male with PS 4 underwent placement of EBS for biliary obstruction with pancreatic cancer (Fig. 2A). At 10 days after ERCP, cholangitis with EBS obstruction occurred; The EBS (across the papilla) was replaced with the plastic stent inside [i.e., above the papilla (Fig. 2B, arrowheads)], and the cholangitis gradually improved. However, at 10 days following stent replacement, he experienced a hospital-acquired influenza infection. Thus, his general condition worsened and he died 14 days after ERCP.

\section{Discussion}

The present study had two findings. First, ERCP for elderly patients with a PS of 3-4, which is the highest risk group, is effective and safe. Second, ERCP in elderly patients with a PS of 3-4 may result in a fatal outcome caused by incidents unrelated to the ERCP procedure.

As regards the first finding, as noted above, ERCP for elderly patients with a PS of 3-4, which is the highest risk group, is effective and safe. While ERCP is a useful procedure for the diagnosis and therapy of pancreatic and biliary disease (14), adverse events of ERCP procedures, such as pancreatitis occasionally result in a fatal outcome. Thus, ERCP procedures for high-risk patients should be performed carefully. It is important to estimate preprocedural risk factors accurately by means of objective clinical tools, applied to elderly patients with comorbidity and possible procedure-related adverse events, to produce satisfactory clinical ERCP outcomes (15). Previously, a number of studies have reported the safety and efficacy of ERCP for elderly patients (2-6). However, it is estimated that the risk factor for ERCP for elderly patients is not only age, but also other clinical conditions. From a geriatric perspective, the assessment of older patients is an interdisciplinary process that should include multiple domains, such as functional and nutritional status, cognition, the accurate evaluation of comorbidity and many others (16). Thus, the assessment of the risk of elderly patients is more complex. Park et al (17) reported that the Duke Activity Status Index (DASI) is a useful predictor for overall serious events related to ERCP. DASI is a valid measure of functional capacity that can be obtained by answering a self-administered questionnaire (18). However, the calculation of DASI is known to be complex. Therefore, an easier index is preferable for the determination of emergent ERCP procedure indications. In the present study, the classification from the Eastern Cooperative Oncology Group (ECOG) PS score was used. The ECOG PS score is a simple and easy method used for the assessment of general well-being, activities of daily life, and tolerance for therapy (7). Elderly patients with depressed PS are considered to be perhaps the highest truly risk group because of the higher prevalence rate of moderate to severe cholangitis and higher CCI, as described later. To our knowledge, this is the first study on the safety and efficacy of ERCP for elderly patients between good and depressed PS groups. 

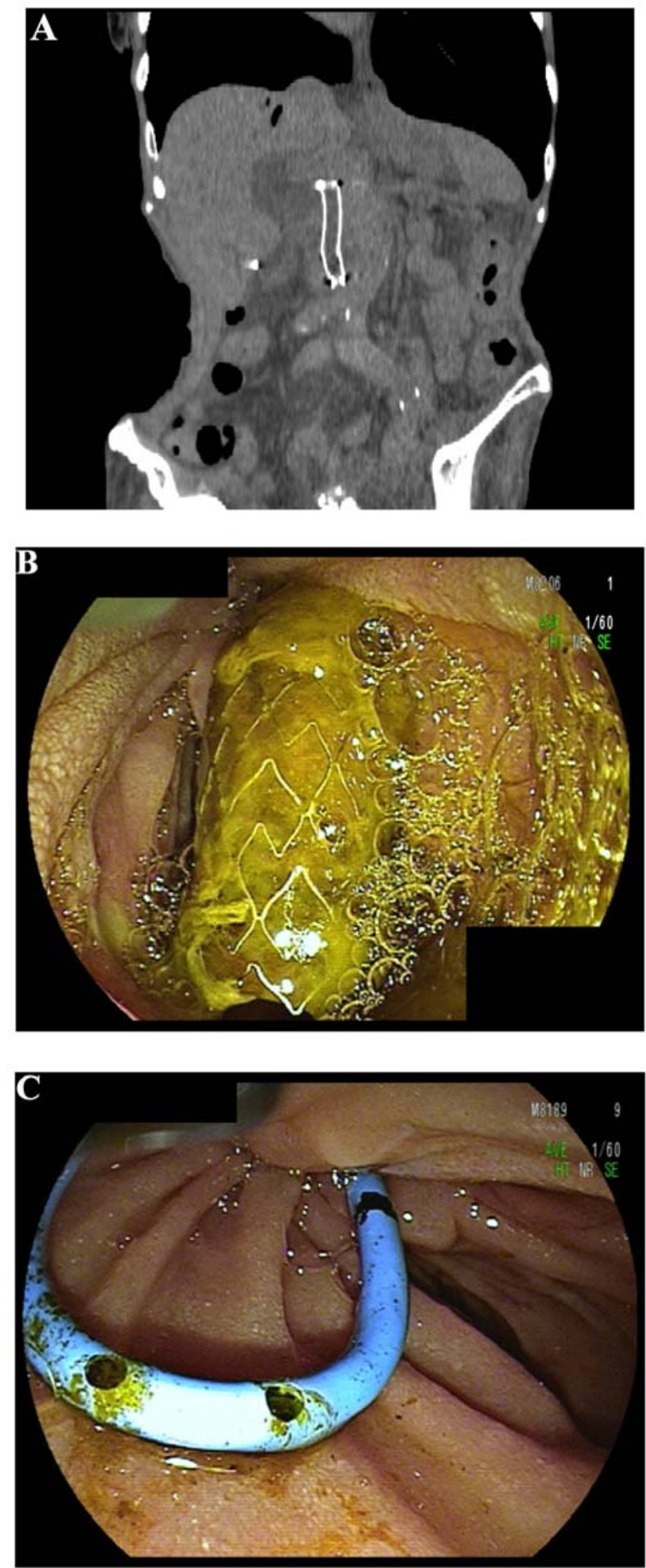

Figure 1. (A) Although SEMS cleaning was performed one month prior, cholangitis by SEMS obstruction recurred. (B) SEMS prolapsed from the common bile duct and was obstructed. (C) EBS was replaced. SEMS, self-expandable metallic stent; EBS, endoscopic biliary stent.

The present study indicated that ERCP is effective and safe even for elderly patients with PS 3-4.

As regards the second finding, ERCP in elderly patients with a PS of 3-4 may result in a fatal outcome caused by incidents unrelated to the ERCP procedure. In the present study, 2
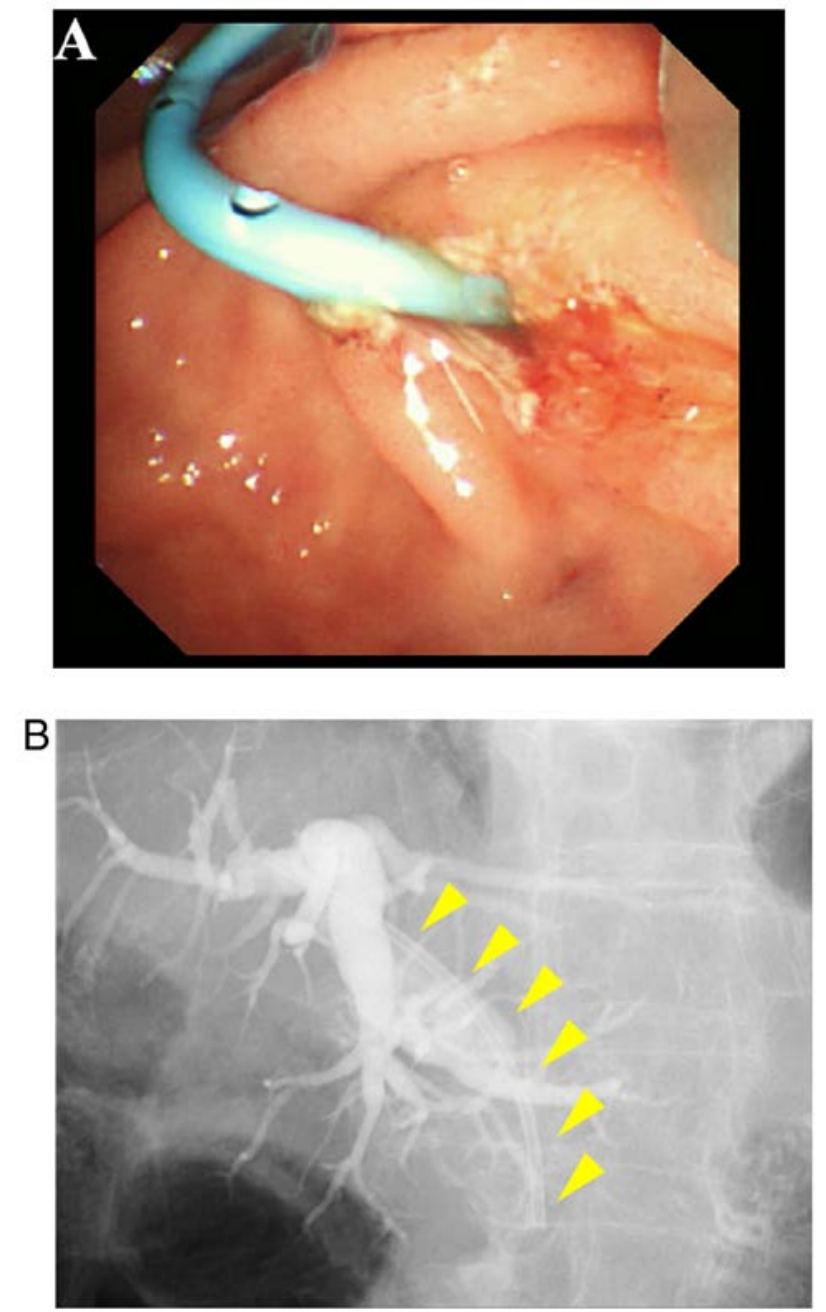

Figure 2. (A) EBS was placed for biliary obstruction with pancreatic cancer. (B) EBS across the papilla was exchanged with the plastic stent inside and above the papilla (arrowheads). EBS, endoscopic biliary stent.

fatal cases occurred during the 30 days following ERCP in the PS 3-4 group, whereas no fatal cases occurred in the PS 0-2 group. One patient's condition was exacerbated by anorexia caused by pancreatic cancer, while another patient's condition was exacerbated by hospital-acquired influenza infection. The general condition of elderly patients with a decreased PS may be easily deteriorated by anorexia or infection due to advanced malignant tumors. Previously, Takahashi et al (19) also reported that patients with a PS of 3-4 were likely to develop poor general conditions, such as aspiration pneumoniae and heart failure following ERCP. Of note, in the present study, both prevalence rates of moderate or severe cholangitis and CCI in the PS 3-4 group were significantly higher than in the PS 0-2 group. CCI is widely used for the assessment of surgery indication or prognosis of other diseases $(9,20-22)$. In other words, a higher CCI indicates vulnerability in the general condition due to several existing diseases. Thus, the general condition of patients in the PS 3-4 group was likely to deteriorate compared to that of the PS 0-2 group in the presence of cholangitis. Moreover, incidents unrelated to the ERCP procedures, such as an influenza infection, may deteriorate the general condition of patients with a PS of 3-4 and may result in a fatal outcome due to a higher CCI. However, the higher 
comorbidity disease rate of the PS 3-4 group with moderate or severe cholangitis indicates that elderly patients with a PS of 3-4 require biliary drainage compared to those with a PS of $0-2$.

As mentioned above, ERCP is effective and safe for elderly patients with not only a PS of 0-2, but also with a PS of 3-4. ERCP procedures should be performed for patients with a PS of 3-4 due to their moderate to severe cholangitis and their vulnerability. However, even if ERCP procedures are successful, careful attention should be paid to the clinical course of elderly patients with a PS of 3-4.

The present study had some limitations. First, it was retrospectively performed in a single center, intended for patients who underwent ERCP for short periods of time. Therefore, the number of elderly patients with a PS of 3-4 was comparatively lower. Additional prospective investigations for more cases are necessary in the future. Second, this study was intended for elderly patients with a PS of 3-4 who underwent ERCP. The clinical course of elderly patients not undergoing ERCP was not investigated, which may be a selective bias. Following this study, it is necessary to investigate the clinical courses of all elderly patients regardless of ERCP. Third, this study did not make comparisons with younger patients. The efficacy and adverse events of ERCP for younger patients with a PS of 3-4 need to be investigated.

Overall, the present study clarified that the technical success rate and complication rate in patients with PS 3-4 were comparable to those of patients with PS 0-2. Thus, ERCP for elderly patients with a PS of 3-4 is effective and safe. However, it should be noted that ERCP in patients with a PS of 3-4 may result in a fatal outcome caused by incidents unrelated to the ERCP procedure.

\section{Acknowledgements}

Not applicable.

\section{Funding}

No funding was received.

\section{Availability of data and materials}

The data of this study are available upon reasonable request. The data are stored at Saiseikai Chuwa Hospital electronic health record system, and the participant data were de-identified in this study.

\section{Authors' contributions}

SA wrote the manuscript. SA, AD, HU, KM and KY were the attending doctors for the patients. SA, AD, HU, KM conducted the ERCP. HY contributed to the conception and design of the study. AD organized the study and the report.

\section{Ethics approval and consent to participate}

This is a retrospective observational study in Saiseikai Chuwa Hospital, not an interventional trial. Thus, the patients in this study were involved within general practice and thus ethics approval and patient consent was not required.

\section{Patient consent for publication}

Not applicable.

\section{Competing interests}

The authors declare that they have no competing interests.

\section{References}

1. Ukkonen M, Siiki A, Antila A, Tyrväinen T, Sand J and Laukkarinen J: Safety and efficacy of acute endoscopic retrograde cholangiopancreatography in the elderly. Dig Dis Sci 61: 3302-3308, 2016.

2. Galeazzi M, Mazzola P, Valcarcel B, Bellelli G, Dinelli M, Pasinetti GM and Annoni G: Endoscopic retrograde cholangiopancreatography in the elderly: Results of a retrospective study and a geriatricians' point of view. BMC Gastroenterol 18: 38 , 2018.

3. Tohda G, Ohtani M and Dochin M: Efficacy and safety of emergency endoscopic retrograde cholangiopancreatography for acute cholangitis in the elderly. World J Gastroenterol 22: 8382-8388, 2016.

4. Yun DY, Han J, Oh JS, Park KW, Shin IH and Kim HG: Is endoscopic retrograde cholangiopancreatography safe in patients 90 years of age and older? Gut Liver 8: 552-556, 2014.

5. Behlül B, Ayfer S, Sezgin V, Altay K, Mustafa C, Cem C, Bilge O, Fatih A, Emrah A, Zafer B, et al: Safety of endoscopic retrograde cholangiopancreatography in patients 80 years of age and older. Prz Gastroenterol 9: 227-231, 2014.

6. Mohammad Alizadeh AH, Afzali ES, Shahnazi A, Sanati A, Mirsattari D and Zali MR: Utility and safety of ERCP in the elderly: A comparative study in Iran. Diagn Ther Endosc 2012: 439320, 2012.

7. Oken MM, Creech RH, Tormey DC, Horton J, Davis TE, McFadden ET and Carbone PP: Toxicity and response criteria of the Eastern Cooperative Oncology Group. Am J Clin Oncol 5: 649-655, 1982.

8. Takada T, Strasberg SM, Solomkin JS, Pitt HA, Gomi H, Yoshida M, Mayumi T, Miura F, Gouma DJ, Garden OJ, et al: TG13: Updated Tokyo guidelines for the management of acute cholangitis and cholecystitis. J Hepatobiliary Pancreat Sci 20: 1-7, 2013.

9. Charlson ME, Pompei P, Ales KL and MacKenzie CR: A new method of classifying prognostic comorbidity in longitudinal studies: Development and validation. J Chronic Dis 40: 373-383, 1987.

10. Miura F, Takada T, Strasberg SM, Solomkin JS, Pitt HA, Gouma DJ, Garden OJ, Büchler MW, Yoshida M, Mayumi T, et al: TG13 flowchart for the management of acute cholangitis and cholecystitis. J Hepatobiliary Pancreat Sci 20: 47-54, 2013.

11. Tanisaka Y, Ryozawa S, Mizuide M, Kobayashi M, Fujita A, Minami K, Kobatake T, Omiya K, Iwano H and Araki R: Usefulness of the 'newly designed' short-type single-balloon enteroscope for ERCP in patients with Roux-en-Y gastrectomy: A pilot study. Endosc Int Open 6: E1417-E1422, 2018.

12. Maple JT, Keswani RN, Hovis RM, Saddedin EZ, Jonnalagadda S, Azar RR, Hagen C, Thompson DM, Waldbaum L and Edmundowicz SA: Carbon dioxide insufflation during ERCP for reduction of postprocedure pain: A randomized, double-blind, controlled trial. Gastrointest Endosc 70: 278-283, 2009.

13. Nakamura K, Yamaguchi Y, Hasue T, Higa K, Tauchi M, Toki M, Sugiyama M and Takahashi S: The usefulness and safety of carbon dioxide insufflation during endoscopic retrograde cholangiopancreatography in elderly patients: A prospective, double-blind, randomized, controlled trial. Hepatogastroenterology 61: 2191-2195, 2014.

14. Adler DG, Baron TH, Davila RE, Egan J, Hirota WK, Leighton JA, Qureshi W, Rajan E, Zuckerman MJ, Fanelli R, et al: ASGE guideline: The role of ERCP in diseases of the biliary tract and the pancreas. Gastrointest Endosc 62: 1-8, 2005. 
15. Mallery JS, Baron TH, Dominitz JA, Goldstein JL, Hirota WK, Jacobson BC, Leighton JA, Raddawi HM, Varg JJ II, Waring JP, et al: Complications of ERCP. Gastrointest Endosc 57: 633-638, 2003.

16. Ellis G, Whitehead MA, O'Neill D, Langhorne $P$ and Robinson D: Comprehensive geriatric assessment for older adults admitted to hospital. Cochrane Database Syst Rev 2011: CD006211, 2011.

17. Park TY, Choi JS, Oh HC, Kim JW, Do JH and Jung YH: Assessment of safety of non-anesthesiologist-assisted endoscopic retrograde cholangiopancreatography based on performance status in elderly patients. J Gastroenterol Hepatol 29: 1943-1948, 2014.

18. Hlatky MA, Boineau RE, Higginbotham MB, Lee KL, Mark DB, Califf RM, Cobb FR and Pryor DB: A brief self-administered questionnaire to determine functional capacity (the Duke Activity Status Index). Am J Cardiol 64: 651-654, 1989.

19. Takahashi K, Nihei T, Aoki Y, Nakagawa M, Konno N, Munakata A, Okawara $\mathrm{K}$ and Kashimura H: Efficacy and safety of therapeutic endoscopic retrograde cholangiopancreatography in patient with native papillae with a performance status score of 3 or 4: A single-center retrospective study. J Rural Med 14: 226-230, 2019.
20. Endo I, Takada T, Hwang TL, Akazawa K, Mori R, Miura F, Yokoe M, Itoi T, Gomi H, Chen MF, et al: Optimal treatment strategy for acute cholecystitis based on predictive factors: Japan-Taiwan multicenter cohort study. J Hepatobiliary Pancreat Sci 24: 346-361, 2017.

21. Ando K, Doi T, Moody SY, Ohkuni Y, Sato S and Kaneko N: The effect of comorbidity on the prognosis of acute lung injury and acute respiratory distress syndrome. Intern Med 51: 1835-1840, 2012.

22. Naito Y, Sasaki H, Takamatsu Y, Kiyomi F and Tamura K: Retrospective analysis of treatment outcomes and geriatric assessment in elderly malignant lymphoma patients. J Clin Exp Hematop 56: 43-49, 2016.

This work is licensed under a Creative Commons Attribution-NonCommercial-NoDerivatives 4.0 International (CC BY-NC-ND 4.0) License. 